Article

\title{
Electrorheological Behavior of Suspensions of Polyimide-Based on the Sodium Salt of 2,5-Diaminobenzenesulfonic Acid
}

\author{
Nikolay Semenov $\mathbb{D}$, Alexander Danilin $\mathbb{D}$, Yulia Karnet $\mathbb{D}$ and Elena Kelbysheva * $\mathbb{D}$ \\ Institute of Applied Mechanics of Russian Academy of Science, 125040 Moscow, Russia; \\ semenov@iam.ras.ru (N.S.); andanilin@yandex.ru (A.D.); iam@iam.ras.ru (Y.K.) \\ * Correspondence: kellena80@mail.ru; Tel.: +7-495-946-1806
}

Received: 14 March 2020; Accepted: 22 April 2020; Published: 29 April 2020

check for updates

\begin{abstract}
Electrorheological suspensions (ERS) of polyimide particles with organoelement fragments from the sodium salt of 2,5-diaminobenzene sulfonic acid were obtained for the first time. Their rheological and electrorheological characteristics, their dependence on the parameters of deformation, and the intensity of the external electric field were studied. It was found that the ERS of PI-Na polyimide particles have a significant electrorheological response. Solid-polyimide materials were previously studied using a scanning electron microscope. The effect of the polyimide concentration on the properties of the solution was studied by spectrophotometry. It was shown that polyimide suspension is a result of increasing intensity as the electric field changes the flow type from Newtonian to pseudoplastic due to polarization of the particles and formation of the chain structures along the power lines of the electric field. The influence of temperature on the change of rheological and electrorheological properties of a polyimide ERS in constant electric fields was investigated.
\end{abstract}

Keywords: electrorheological suspensions; viscosity; adaptive materials; elastomers; electric field controlled materials; metal-containing polyimide; salt sulfoacid; polymer

\section{Introduction}

Electrorheological suspensions (ERS) are materials whose rheological properties, such as viscosity, yield strength, shear modulus, etc. [1], change under the influence of an external, electric field. When an electric field is applied, the ERS switch from a low viscosity and a liquid, flowing state to a solid or viscoplastic state. Typically, these changes are reversible and occur within milliseconds. Therefore, ERSs are very promising from a practical point of view.

Due to their unique properties, ERSs are considered "smart materials" [2], whose properties can be adjusted as needed for various practical tasks. For example, ERSs are used as working bodies in electrically controlled mechanical devices, such as controlled dampers, electromagnetic clutches, various vibration protection devices, etc. [3,4]. A number of applications for ERSs in space technology, biomechanics, biomedicine, etc., are proposed in [5].

ERSs are suspensions of polarized solid particles in a nonconductive oil [6]. In the external electric field, the particles are polarized and form chain structures along electric field lines. ERSs, on the basis of polymers [2,7], and in particular, polyimides [8,9] are among the most frequently studied. Polyimide chains are often easy to polymerize, and various aromatic diamines and dianhydrides of acids are commercially available. For practical purposes, it is important to select such polyimide structures that are highly soluble, nonhydrolyzable, and can be used as cast films. Polyimides have a high degree of aromaticity and enable the efficient formation of charge transfer complexes (CTC) for both intramolecular and intermolecular charge transfers, which are necessary for strong electrorheological responses. 
It is important to create a material whose properties can be easily adjusted by entering several functional fragments, which can either by themselves or through further modification can dramatically change the properties of the material. It is known that the presence of functional groups containing fluorine reduces the formation of CTC significantly in polyimides and weakens the charge transfer. Also, the materials based on polyimide polymers with the presence of fluorinated side groups are known to have unusual thermomechanical behavior [10]. Only a few examples of metal-containing polyimides have been reported. Such materials are both difficult to make and to characterize, and their properties are not easy to study [11]. Despite these difficulties, polyimides containing metal ions in the main chain were obtained for the first time in reference [12].

Previously, we obtained polyimides that have in the polymer chain a polar and chemically active sulfo group, which is useful for injecting new atoms or groups [13]. In this paper, a new material containing polyimide with $\mathrm{Na}$ salt salt-acid fragments and fluorinated groups in the side chain as the dispersed phase is obtained. It is shown that this material has a powerful electrorheological response, which opens the possibility of creating controlled damping devices based on it.

\section{Materials and Methods}

Tensor 37 (Bruker) IR spectrometer (Bruker Optik GmbH, Ettlingen, Germany) was used to detect infrared (IR) spectra with the resolution of $2 \mathrm{~cm}^{-1}$ in $\mathrm{KBr}$ cells. The UV/Vis spectra were recorded with a Specord M40 spectrophotometer (Carl Zeiss Industrielle Messtechnik GmbH, Oberkochen, Germany). NMR ${ }^{1} \mathrm{H}$ spectra (internal standard - solvent, from $\mathrm{Me}_{4} \mathrm{Si}, \mathrm{DMSO}-\mathrm{d}_{6}-2.50 \mathrm{ppm}$ ) were recorded with a "Bruker Avance 400" spectrometer (400.13 and 100.61 MHz, respectively, Bruker Optik GmbH, Ettlingen, Germany). Rheostress RS 150 rheoviscosimeter (Thermo Haake GmbH, Karlsruhe, Germany) with a plane-to-plane measuring node was used to measure the electrorheological properties. The scanning electron microscope, JSM-6480hv (JEOL Ltd, Akisima, Japan), was used to examine the polyimide sample. Photos of the samples were taken using the Altami MET 5T optical microscope (Altami, St. Petersburg, Russia). The particle-size distribution was analyzed by dynamic-light scattering on a Nanobrook OMNI-BNDL (Brookhaven Instruments, New York, NY, USA) with a laser wavelength of $637 \mathrm{~nm}$. For the synthesis of polyimides, the following reagents were used: 2,5-diaminobenzene sulfonic acids, 4,4' - (hexafluorisopropylidene)diphthalic anhydride, mesitylene, and dimethylformamide (DMF) (all reagents and solvents: Sigma-Aldrich Chemie GmbH, Munich, Germany), PMS-400 (Penta-92, Moscow, Russia). They are commercially available and were used without additional purification.

\section{Results and Discussion}

\subsection{Synthesis and Characterization of Polyimide}

PI-Na polyimide was prepared by a single-stage, high-temperature polycondensation in solution [14] of the sodium salt of 2.5-diaminobenzene sulfonic acid (1-Na) and 4.4'-(hexafluorisopropylidene) diphthalic anhydride (2) (Scheme 1).

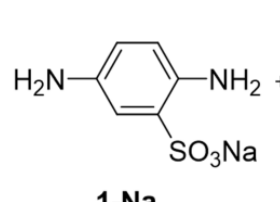

$1-\mathrm{Na}$

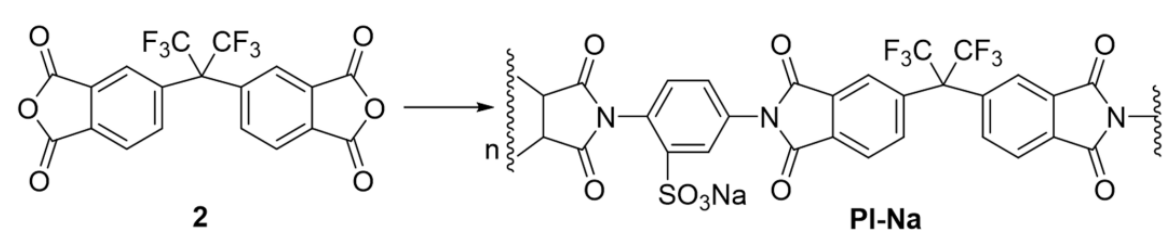

Scheme 1. Synthesis of polyimide PI-Na.

Sodium salt 1-Na was obtained by the method described in [15]. $\mathrm{NaHCO}_{3}(2.2 \mathrm{~g}, 26.6 \mathrm{mmol})$ was added to a solution of 2.5-diaminobenzene sulfonic acid $(5.0 \mathrm{~g}, 26.6 \mathrm{mmol})$ in $30 \mathrm{~mL}$ of deionized water 
and $20 \mathrm{~mL}$ of acetone in portions. The solution was heated until the water was completely removed, and the remaining substance was vacuum-dried at $90^{\circ} \mathrm{C}$ for $4 \mathrm{~h}$. The yield was of $\mathbf{1}-\mathrm{Na} 5.5 \mathrm{~g}(98 \%)$. NMR ${ }^{1} \mathrm{H}$ was $\left(400 \mathrm{MHz}, 25^{\circ} \mathrm{C}\right.$, DMSO-d $\left.\mathrm{d}_{6}\right): 4.24\left(\mathrm{~s}, 2 \mathrm{H}, \mathrm{NH}_{2}\right), 4.80\left(\mathrm{~s}, 2 \mathrm{H}, \mathrm{NH}_{2}\right), 6.36(\mathrm{~m}, 2 \mathrm{H}, \mathrm{H}-\mathrm{Ph})$, and 6.84 (br.s., 1H, H-Ph). IR spectrum (KBr) and $v / \mathrm{cm}^{-1}: 3408,3383,3322 v(\mathrm{NH}), 1182$, and $1028 v(\mathrm{SO})$.

Predictability of the impurity composition, a high degree of imidization, and the thermal and chemical stability of the polymer are the main requirements to a method for making polymer material.

$\mathrm{X}$-ray fluorescence elemental analysis data confirmed the elemental composition of the resulting polyamide (Table 1).

Table 1. Thermal properties and elemental analysis data of the PI-Na.

\begin{tabular}{|c|c|c|c|c|c|c|c|c|c|}
\hline Polyimide & Formula & $T_{\mathrm{g}}\left({ }^{\circ} \mathrm{C}\right)$ & & & Eleme & al Ana & is $(\%)$ & & \\
\hline \multirow{3}{*}{ PI-Na } & \multirow{3}{*}{$\mathrm{C}_{25} \mathrm{H}_{9} \mathrm{O}_{7} \mathrm{~F}_{6} \mathrm{NaN}_{2} \mathrm{~S}$} & \multirow{3}{*}{420} & & $\mathrm{C}$ & $\mathbf{H}$ & $\mathbf{N}$ & F & S & $\mathrm{Na}$ \\
\hline & & & Calcd & 48.56 & 1.47 & 4.53 & 18.44 & 5.18 & 3.71 \\
\hline & & & Found & 47.99 & 2.27 & 5.13 & 17.01 & 4.36 & 3.50 \\
\hline
\end{tabular}

The IR spectrum shown in Figure 1 confirms the structure of Na-containing polyimide PI-Na. The IR data exhibits characteristic-absorption bands corresponding to the deformation oscillation of the image ring $(\mathrm{C}-\mathrm{N})$ located in the region of $720 \mathrm{~cm}^{-1}$, as well as the peaks at 1782 and $1720 \mathrm{~cm}^{-1}$, which correspond to symmetrical and asymmetric oscillations of the imide carbonyl groups $(\mathrm{N}-\mathrm{C}=\mathrm{O})$. A peak in the area of $1026 \mathrm{~cm}^{-1}$ and a sharp peak in the area of $1099 \mathrm{~cm}^{-1}$ are assigned to the $\mathrm{SO}_{2}$ group. The bands in the area of 1366 and $1207 \mathrm{~cm}^{-1}$ correspond to the vibrations of the C-F group. It should be noted that the polyimide PI-Na is quite hygroscopic and requires predrying before the instrumental analysis.

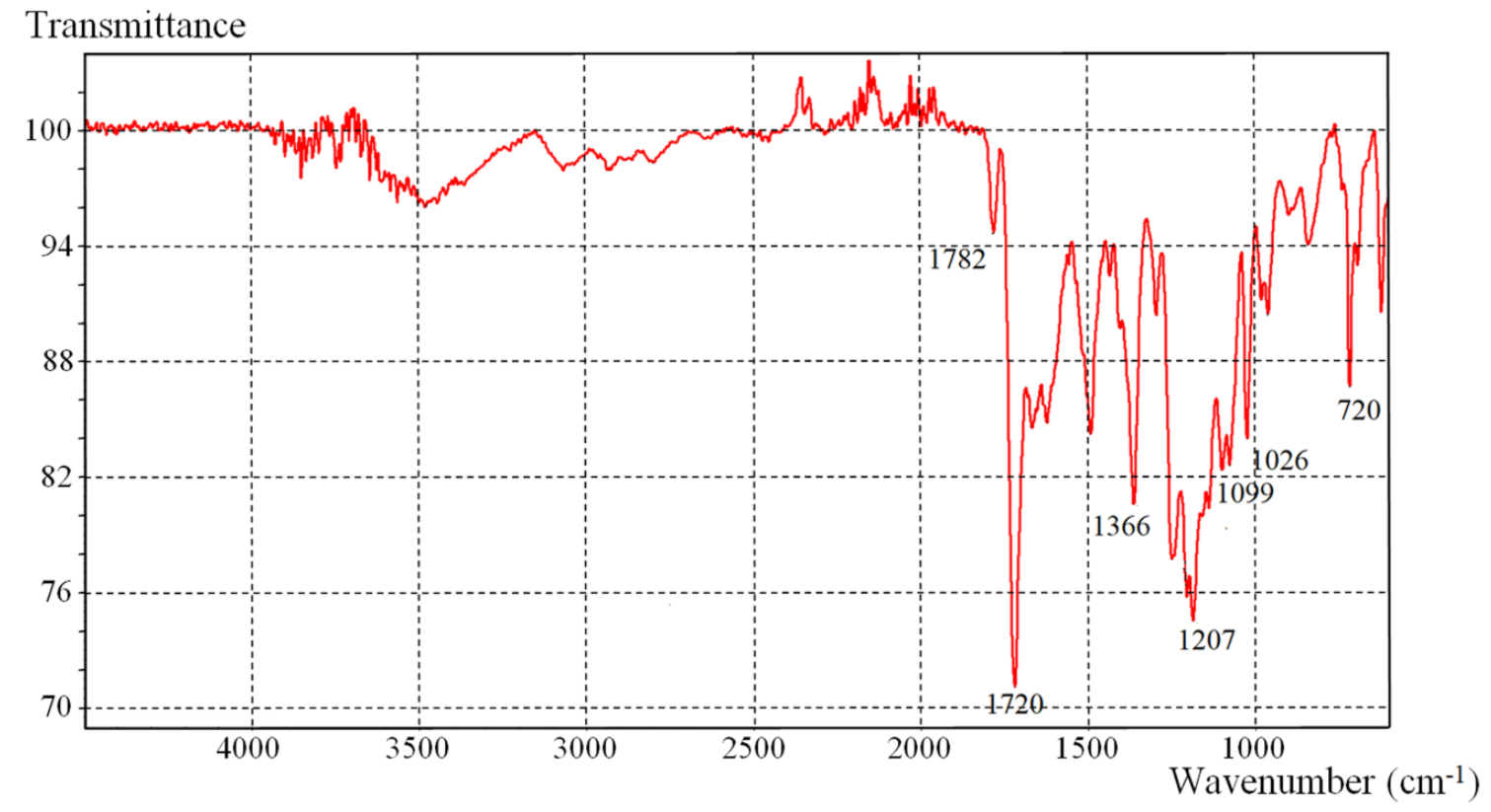

Figure 1. Infrared (IR) spectra of PI-Na.

Analysis of solution PI-Na by dynamic-light scattering shows that the studied polymers are polydisperse systems. The size of the studied particles in the solution mainly lies in the range from 100 to $295 \mathrm{~nm}$ in DMF.

UV-visible spectra (Figure 2) of polyimide solutions reveal the influence of the solution concentration on their properties. As shown in Figure 2, reducing the concentration of the solution by four times leads to a hypsochromic (or blue) shift from 306 to $290 \mathrm{~nm}$. Likely, this is due to the 
presence of intermolecular interactions between segments of polyimides in the solution. The higher the concentration of the solution, the smaller the distance between the segments, and the interaction between them increases. This is consistent with the literature data in [16].

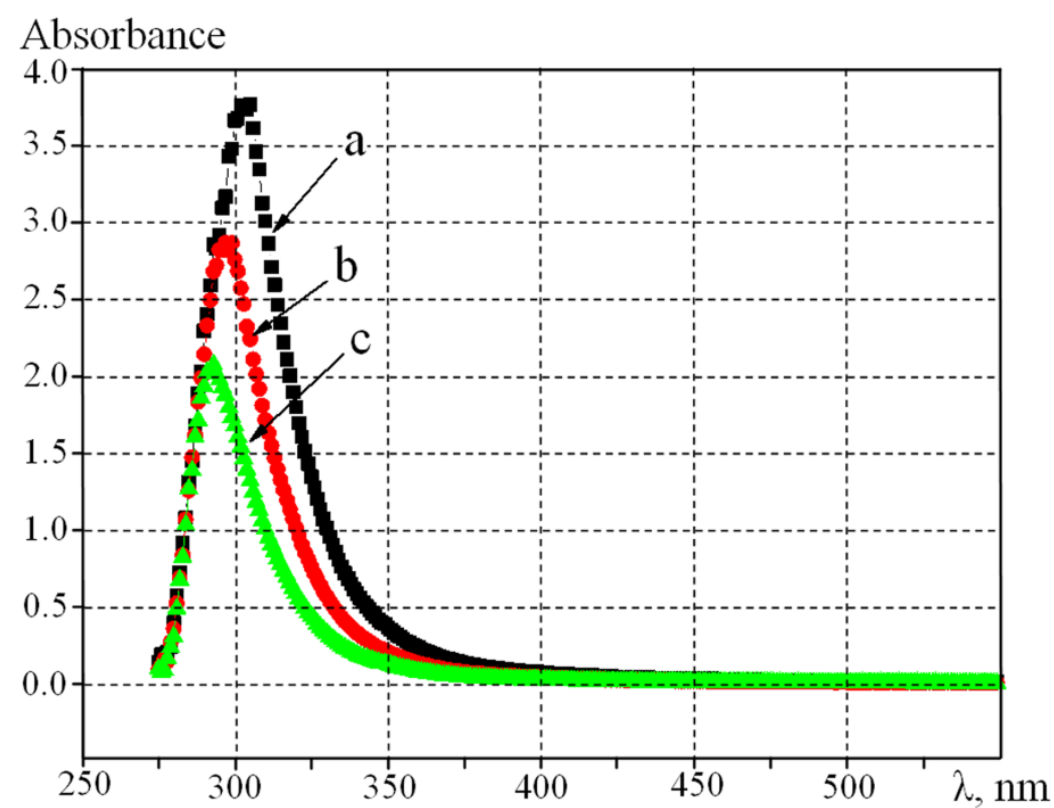

Figure 2. UV-visible spectra of polyimide solutions: (a) $1 \%$, (b) $0.5 \%$, and (c) $0.25 \%$ in dimethylformamide (DMF).

The surface morphology of the material was studied with electron microscopy. These images (Figure 3) show that the polyimide is a large crystallite with a nonuniform layered structure. The results of X-ray diffraction analysis of the surface are shown in Table 2.
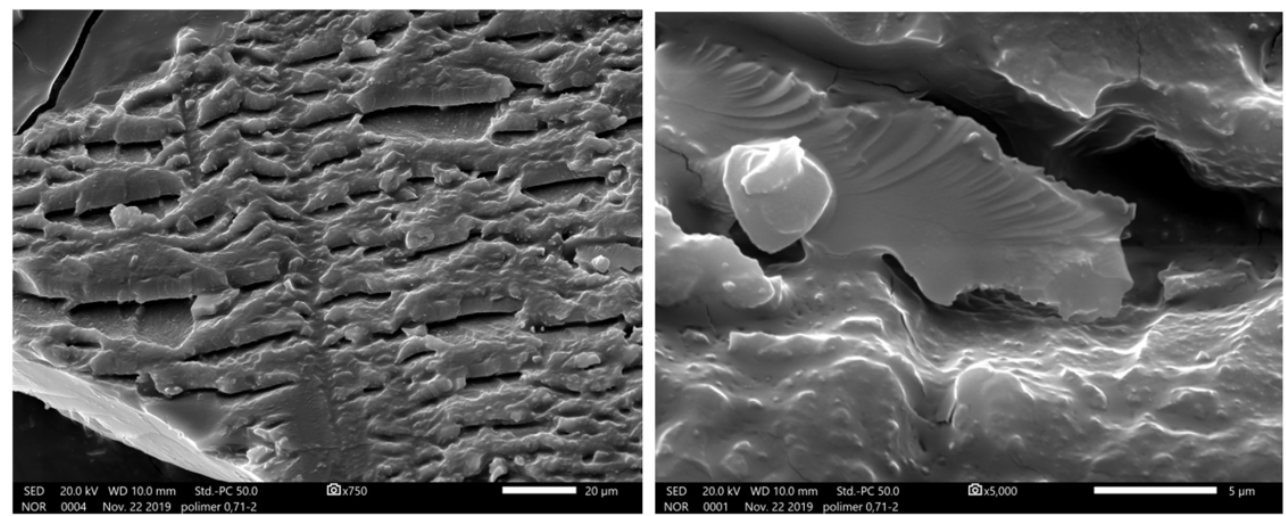

Figure 3. Electron-microscope images taken at different locations of the polyimide-material surface: center, $750 \times($ left $)$ and edge, 5000× (right).

Table 2. Data quantitative X-ray microanalysis for the surface of PI-Na-based material obtained using REM.

\begin{tabular}{cccccccc}
\hline Polyimide & Formula & \multicolumn{7}{c}{ Analysis (\%) } \\
\hline \multirow{2}{*}{ PI-Na } & $\mathrm{C}_{25} \mathrm{H}_{9} \mathrm{O}_{7} \mathrm{~F}_{6} \mathrm{NaN}_{2} \mathrm{~S}$ & $\mathbf{C}$ & $\mathbf{O}$ & $\mathbf{N}$ & $\mathbf{F}$ & $\mathbf{S}$ & $\mathbf{N a}$ \\
\cline { 3 - 8 } & & 39.27 & 17.70 & 8.61 & 21.06 & 7.49 & 5.88 \\
\hline
\end{tabular}




\subsection{Electrorheological Experiments}

Previously, we studied the electrorheological properties of materials based on polyimide particles of different structures, such as PMDA-APS and BTDA-ODA or BTDA-APB [17]. It was shown that polyimides with an articulated bond of polymer's chain exhibit a large ER effect. Furthermore, polyimide particles with some structure exhibited a negative ER effect, e.g., on BTDA-APB. This was an effect where the viscosity drops while the electric field strength increases. This was also be observed using optical microscopy. When applying an electric voltage to ER, polyimide particles showing negative electrorheological effects did not move towards power lines and did not create structured chains, but started rotating around their axis with precession [17] or separating on the cathode. Entering the polar-sulfonic group [13] into a polyimide structure allowed creating polymers without articulated bonds with a direct ER effect. It also opened the extended possibilities for functional modifications. Specifically, the creation of organometallic complexes and salts. New materials began to show a direct ER effect under an applied electrical field due to polyimide particles moving towards the power lines of the electric field strength. We suggested that protons in $\mathrm{SO}_{3} \mathrm{H}$ are mobile and are distributed between acid centers; thus, creating a partial charge in the molecule. This, in turn, led to a dramatic change in the mechanical properties of electrorheological suspensions. At the same time, the sulfo group was useful for additional adjustments of required properties in new materials. In this work, the electrorheological properties of polyimides with modified sulfonic acid fragments were firstly obtained and studied.

Microscopic photo images of suspensions with different concentrations, with and without the presence of the electric field, are shown in Figure 4.

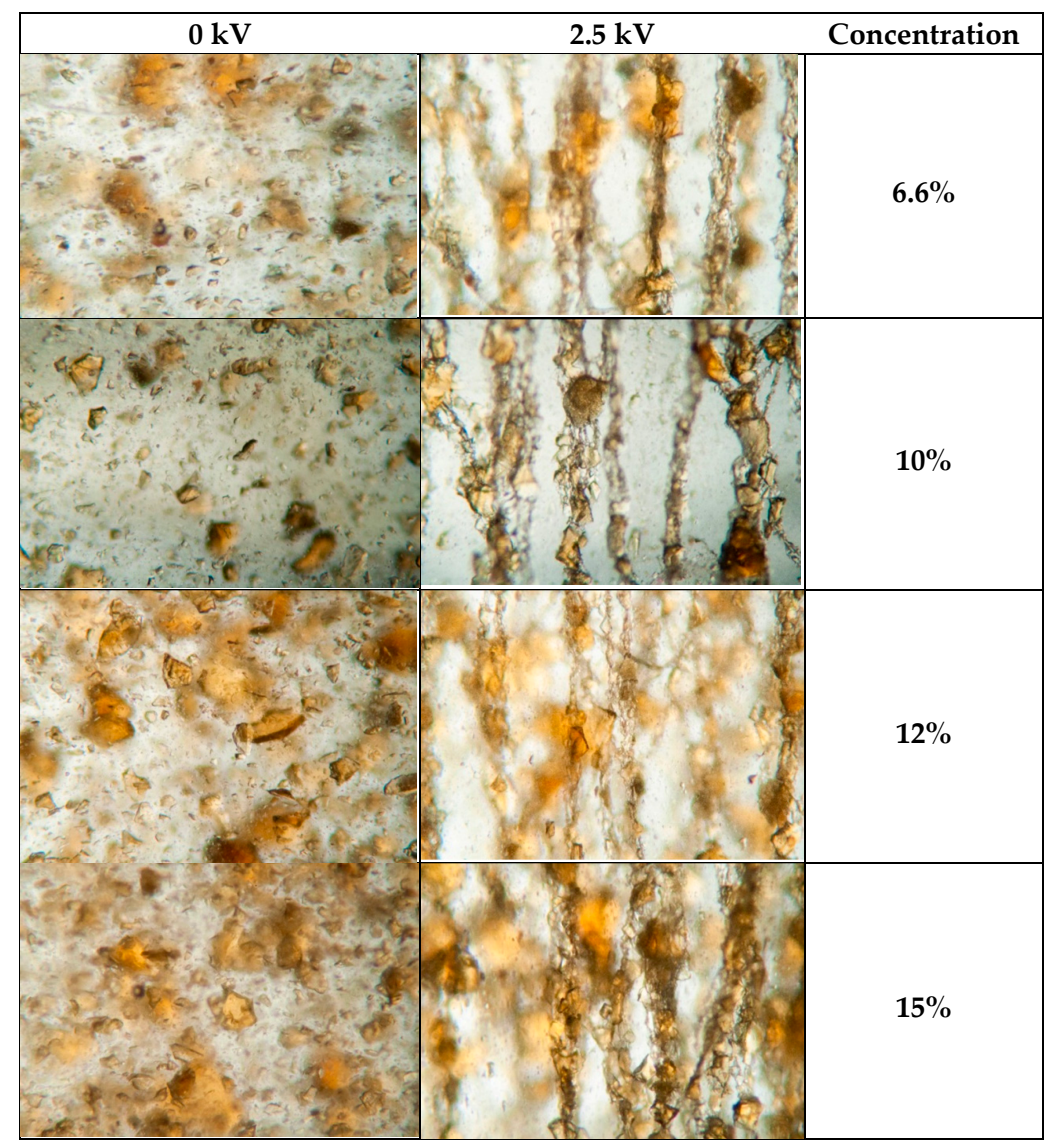

Figure 4. Micrographs of suspensions of polyimides without a field (left) and with a field of $2.5 \mathrm{kV}$ (right) (scale $1 \mathrm{~mm}$ by the height of the picture). 
Figure 4 shows that the PI-Na suspension particles in the absence of an electric field are in the form of unevenly dispersed suspensions, with solid-phase particles of different sizes. After applying the electric field, the polyimide particles line up along the field lines of the electric field, forming thread-like structures. There are always four such structures per millimeter of the analyzed length in the transverse direction to the electric field, regardless of the concentration.

\subsubsection{Electrorheology Characteristics}

In this study, electrorheological tests were conducted on the plane-plane operating unit. An ER unit is a system of flat, stainless steel electrodes (working surface diameter $35 \mathrm{~mm}$ ) isolated from the onboard viscometer systems by a ceramic axis. The top electrode (a flat disc) was fixed on an axle connected to the step drive that generates different speeds of rotation (revolutions). The second electrode was also disk-shaped, immobile, and was located under the first. The mutual position of work surfaces was rigidly fixed with a special structure. To ensure a uniform clearance between the flat surfaces $(1 \mathrm{~mm})$, the distance between them was carefully calibrated. The voltage applied to the cell was fed via a slider contact to the bearing, which was connected to the metal axle of the upper electrode and the lower fixed electrode. Electrorheological experiments were performed in the mode of a steady shear flow, to which the electric field was applied in the normal direction in the voltage range from 0 to $4 \mathrm{kV}$. Shear velocity change interval in these studies ranged from 0.1 to 200 and $30 \mathrm{~s}^{-1}$ in the experiment with constant stress. The temperature was controlled by a liquid-thermos controller with $0.1^{\circ} \mathrm{C}$ accuracy on the low plate.

Rheological measurements were performed with a gradually increasing shear-strain rate from 0.1 to $200 \mathrm{~s}^{-1}$ at the temperature of $20^{\circ} \mathrm{C}$ for $10 \%$ by weight of PI-Na polyimide suspensions. The results are shown in Figures 5 and 6.

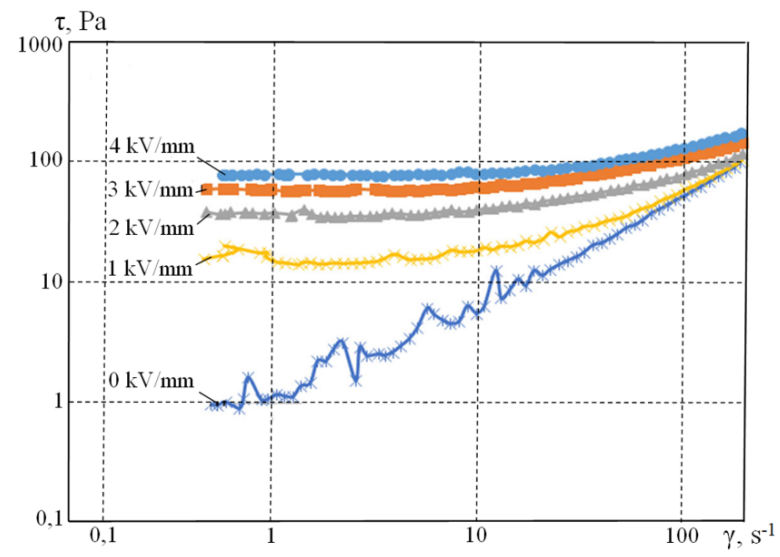

Figure 5. The dependence of stress versus the shear rate of the suspension PI-Na.

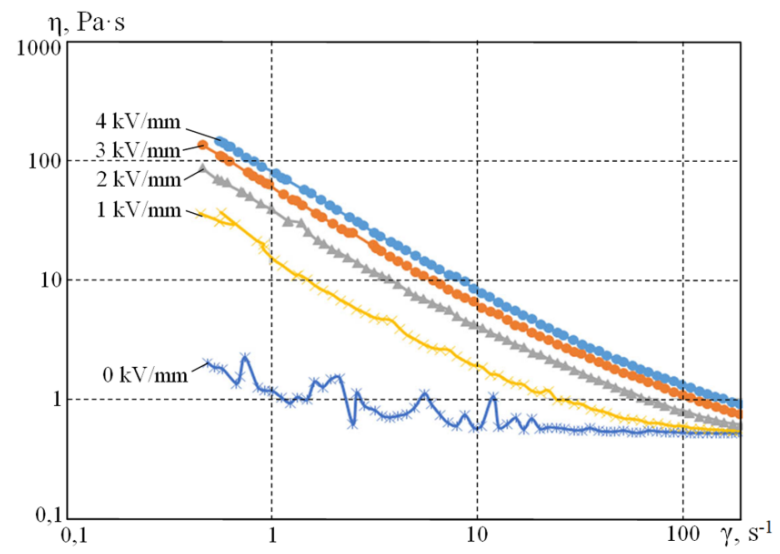

Figure 6. The dependence of viscosity on the shear rate of the suspension PI-Na. 
Figures 4 and 5 show that the electric field changes the suspension-flow pattern from linear (Newtonian) to viscous (Ostwald-de Ville). For a given shear rate, the viscosity of the suspension increases with the increase in the applied electric voltage, which is typical for electrorheological suspensions. With the increase in the shear rate, the structural bonds of the filler do not have enough time to form, and the value of viscosity converges to that of without the field.

\subsubsection{The Influence of Concentration}

Suspensions with solid-phase concentrations of $6.6 \%, 10 \%, 12 \%$, and $15 \%$ by weight were investigated. As the increase in the concentration of solid particles leads to the increase of initial viscosity of the suspension, a direct comparison of the flow curves is not suitable for comparison of the effect of the applied electric field at different concentrations. The relative parameter of the given value of tangent stresses: $\Delta \tau / \tau_{0}=\left(\tau-\tau_{0}\right) / \tau_{0}$ is more appropriate. Here $\tau$ is the tangent stress, and $\tau_{0}$ is the tangent stress at zero electric field strength.

Figure 7 shows the values of the shear stresses measured at a constant shear rate of $30 \mathrm{c}^{-1}$ and a temperature of $20^{\circ} \mathrm{C}$ as a function of voltage, which varied from 0 to $4 \mathrm{kV} / \mathrm{mm}$.

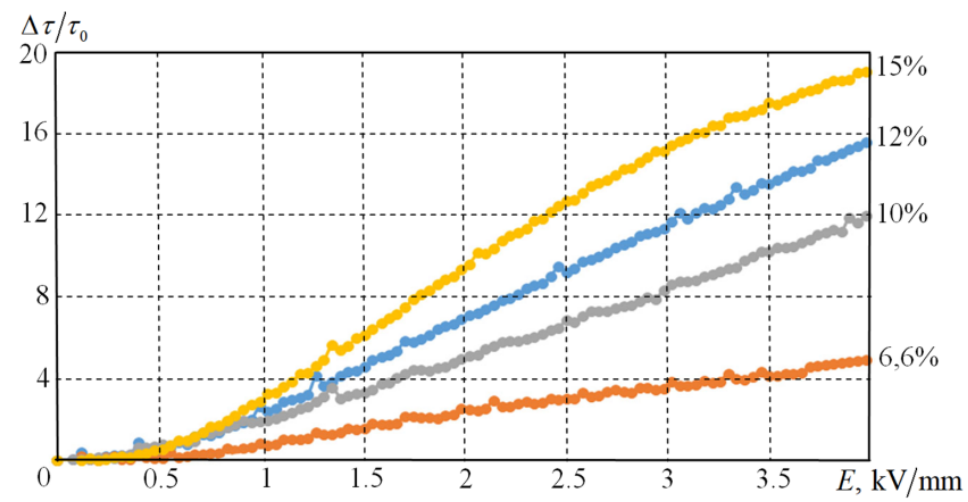

Figure 7. The dependence of the adjusted value of shear stresses on the electric field at $20{ }^{\circ} \mathrm{C}$.

Our results clearly demonstrate that the increase in concentration leads to an increase in the ER effect. For the suspension with the $6.6 \%$ of particles by mass, the tangential stresses show a four-fold increase at $4 \mathrm{kV} / \mathrm{mm}$, and a nearly 20 -fold increase for the $15 \%$ suspension.

\subsubsection{The Influence of Temperature}

Figure 8 shows shear stress at a constant shear rate as a function of the applied voltage in the range between 0 and $4 \mathrm{kV}$ for several temperatures between 20 and $50{ }^{\circ} \mathrm{C}$.

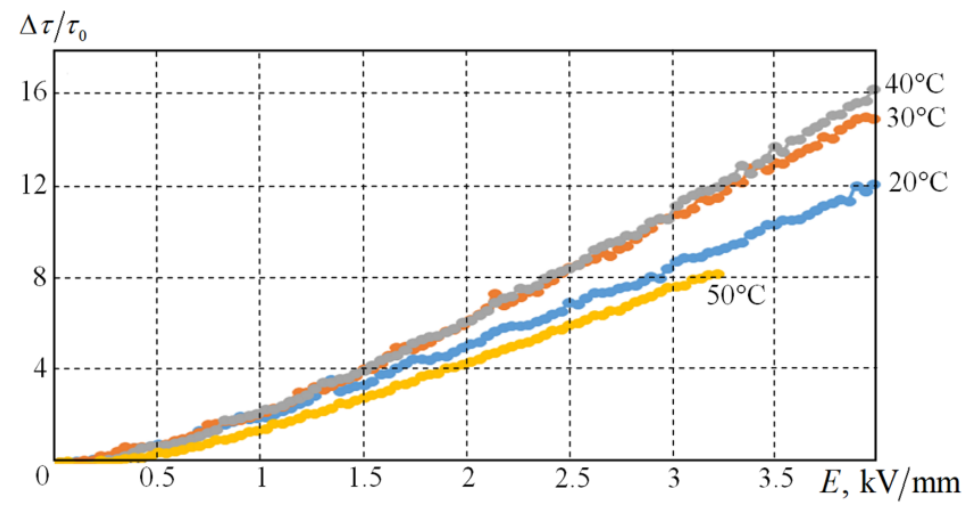

Figure 8. The dependence of the adjusted value of shear stresses on the electric field in temperatures $20,30,40$, and $50{ }^{\circ} \mathrm{C}$ for suspensions of concentration of polyimide PI-Na $10 \mathrm{wt} \%$. 
At $50{ }^{\circ} \mathrm{C}$ and the voltage of $\sim 3 \mathrm{kV} / \mathrm{mm}$, a short circuit occurs in the measuring node, and the current increases abruptly. Apparently, this is due to the presence of sodium ions, which leads to the appearance conductivity in the suspension.

As the viscosity of the medium decreases with an increase in temperature, a direct comparison of flow curves is inconvenient for evaluating the effect of temperature. A comparison of the area between the flow curves is much more informative. It is well known that the area under the flow curves represents the energy $\left(\mathrm{W} / \mathrm{m}^{3}\right)$ required for deforming a single volume of the sample $[18,19]$. The area between the curves represents the energy that is required to destroy the filaments of the filler. Therefore, comparing the areas under the flow curves at different temperatures is convenient for representing the contribution of oriented chains to the changes in rheological properties. Let us denote the areas under the flow curves in the absence of voltage and in its presence by $S_{0}$ and $S_{i}$, respectively. Let us also introduce the value of the area difference $\Delta S=S_{i}-S_{0}$, as shown in Figure 9 at $20^{\circ} \mathrm{C}$ and $4 \mathrm{kV}$. Then the ratio $\Delta S / S_{0}$ shows how many times more or less energy needs to be spent on the deformation of the sample in the applied electric field compared to the deformation process without a field.

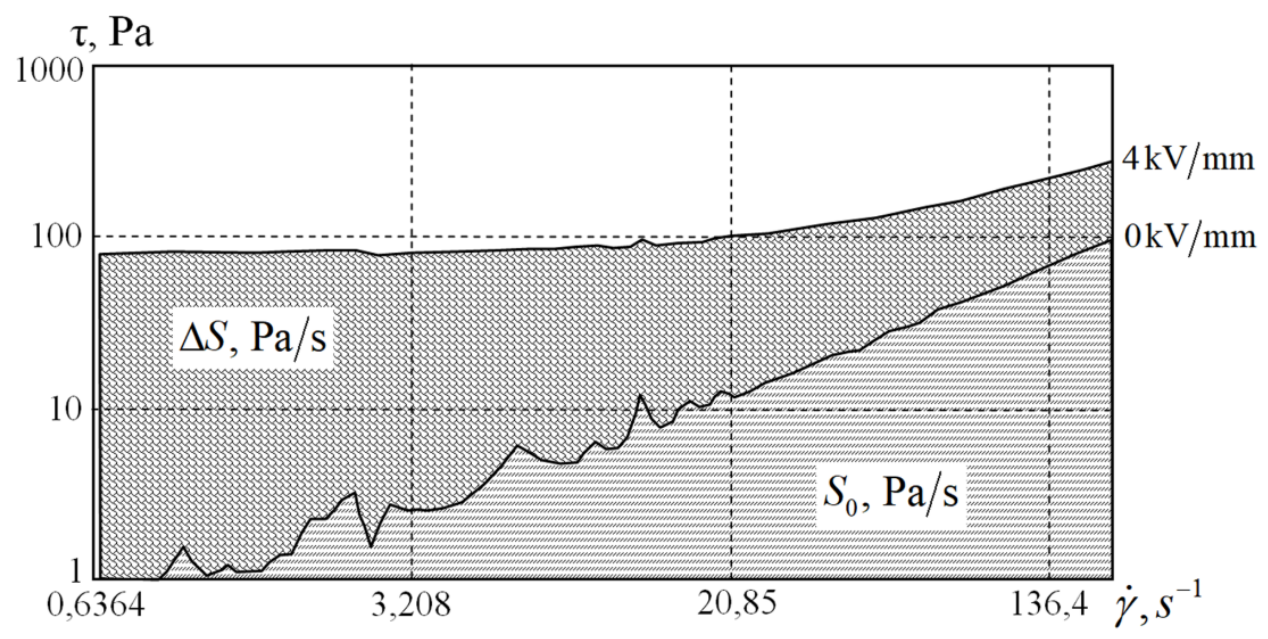

Figure 9. The difference of area under a flow curve for a $10 \mathrm{wt} \%$ suspension of polyimide PI-Na at $20^{\circ} \mathrm{C}$ and 0 and $4 \mathrm{kV}$.

Figure 10 shows the dependence of $\Delta S / S_{0}$ versus the electric field strength. It can be seen that when the temperature increases, the relative energy cost for the deformation of the sample increases. This is because the viscosity of the medium decreases with temperature, the forces of interaction between the electrorheological particles remain the same.

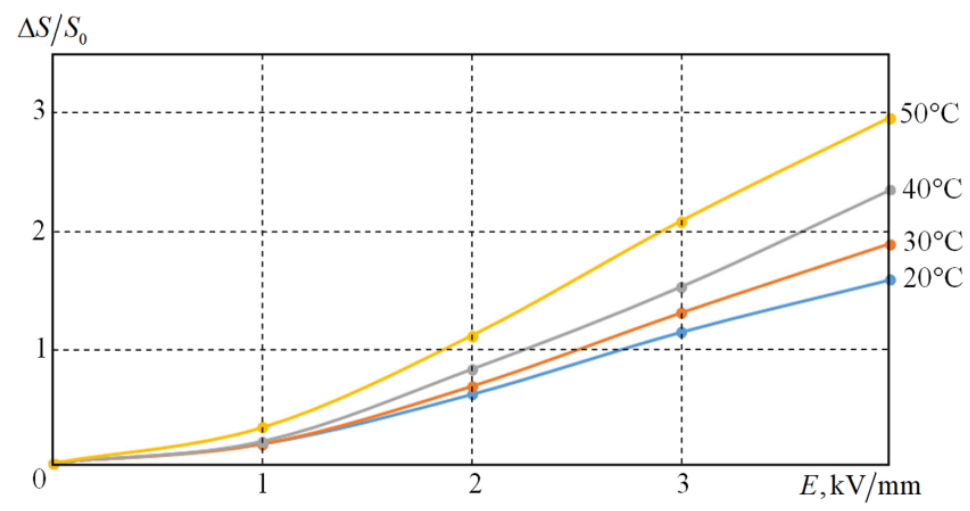

Figure 10. The dependence of $\Delta S$ on the electric field in temperatures $20,30,40$, and $50{ }^{\circ} \mathrm{C}$. 


\section{Conclusions}

In this work, suspensions of polyimide particles with metal-containing fragments were obtained for the first time. Their rheological and electrorheological characteristics, their dependence on the parameters of deformation, and the intensity of the external electric field were studied. It was found that the ERS of PI-Na polyimide particles has a significant electrorheological response. It was shown that increasing the concentration of filler particles in the suspension leads to an increase in the ER effect. Thus, at $6.6 \%$ by mass of particles in the suspension by mass, shear stresses increased four-fold at $4 \mathrm{kV} / \mathrm{mm}$, and at $15 \%$, the 20 -fold increase was observed. The influence of temperature changes on the rheological and electrorheological properties of polyimide ERSs in constant electric fields was demonstrated. It was shown that as the temperature increased, the viscosity of the medium fell, but the structure of the sample did not change. Thus, the change in electrorheological properties and electrorheological response can be most efficiently achieved by the simultaneous effect of the electric field and temperature. Thus, our data should be taken into account for designing devices that use ERSs in conditions when they are subject to heating during operation.

Author Contributions: E.K.: resources, investigation, visualization, writing original draft. A.D.: conceptualization, methodology, writing, reviewing, and editing. Y.K.: data curation, project administration, and supervision. N.S.: software, investigation, and formal analysis. All authors have read and agreed to the published version of the manuscript.

Funding: This research was funded by Ministry of Science and Higher Education of the Russian Federation, ID theme AAAA-A19- 119012290118-3 and AAAA-A19-119012290177-0.

Acknowledgments: The investigations of polyimides powders were performed using SEM of the Collective Use Center "Materials Science and Metallurgy" of NUST MISiS.

Conflicts of Interest: The authors declare no conflict of interest.

\section{References}

1. Block, H.; Kelly, J.P.; Qin, A.; Watson, T. Materials and Mechanisms in Electrorheology. Langmuir 1990, 6, 6-14. [CrossRef]

2. Filisko, F.E. Current developments in electrorheological materials. In Smart Mater; Schwartz, M., Ed.; CRC Press: Boca Raton, FL, USA, 2008; pp. 21.1-21.6. ISBN 9781420043723.

3. Bullough, W.A.; Johnson, A.R.; Hosseini-Sianaki, A.; Makin, J.; Firoozian, R. Electro-rheological clutch: Design, performance characteristics and operation. Proc. Inst. Mech. Eng. Part I J. Syst. Control Eng. 1993, 207, 87-95. [CrossRef]

4. Choi, S.B.; Lee, D.Y. Rotational motion control of a washing machine using electrorheological clutches and brakes. Proc. Inst. Mech. Eng. Part C J. Mech. Eng. Sci. 2005, 219, 627-637. [CrossRef]

5. Hao, T. Electrorheological Fluids: The Non-Aqueous Suspensions; Mobius, P., Miller, R., Eds.; Elsevier Science: Amsterdam, Netherlands, 2006; ISBN 9780444521804.

6. Hao, T. Electrorheological suspensions. Adv. Colloid Interface Sci. 2002, 97, 1-35. [CrossRef]

7. Kim, J.W.; Kim, S.G.; Choi, H.J.; Suh, M.S.; Shin, M.J.; Jhon, M.J. Synthesis and electrorheological characterization of polyaniline and Na+-montmorillonite clay nanocomposite. Int. J. Mod. Phys. B 2001, 15, 657-664. [CrossRef]

8. Yanovsky, Y.G.; Nikitina, E.A.; Karnet, Y.N.; Nikitin, S.M. Smart materials with electrically controlled properties. Electrorheological suspensions with a nanosized polymeric dispersed phase part 1. quantum-mechanical modeling of the electrorheological effect. Int. J. Nanomech. Sci. Technol. 2011, 2, 145-165. [CrossRef]

9. Yanovsky, Y.G.; Semenov, N.A.; Kelbysheva, E.S.; Karnet, Y.N. Electrorheological suspension with a nanosized polymeric dispersed phase significance and role of double dielectric layer. Compos. Mech. Comput. Appl. 2015, 6, 75-86. [CrossRef]

10. Jung, Y.; Yang, Y.; Lee, S.; Byun, S.; Jeon, H.; Cho, M.D. Characterization of fluorinated polyimide morphology by transition mechanical analysis. Polymer 2015, 59, 200-206. [CrossRef]

11. Achar, B.A.; Fohlen, G.M.; Parker, J.A. Heat-resistant poly(metal phthalocyanine)imide copolymers using benzenetetracarboxylic dianhydride. J. Polym. Sci. Polym. Chem. Ed. 1985, 23, 801-811. [CrossRef]

12. Zeng, W.; Qiu, W.; Liu, J.; Yang, X.; Lu, L.; Wang, X.; Dai, Q. Synthesis and characterization of polyimides from metal-containing (Ba, $\mathrm{Sr}, \mathrm{Pb}, \mathrm{Zn})$ diamines. Polymer 1995, 36, 3761-3765. [CrossRef] 
13. Danilin, A.; Kydralieva, K.; Semenov, N.; Kelbysheva, E. Electrorheological properties of polyimide nanoparticles suspensions. Mater. Today Proc. 2020. [CrossRef]

14. Semenov, N.A.; Kelbysheva, E.S.; Vlasov, A.N.; Rabinskii, L.N. Polyimides exhibiting a negative electrorheological response. Russ. Metall. 2017, 2017, 1103-1108. [CrossRef]

15. Hasheminasab, A.; Engle, J.T.; Bass, J.; Herrick, R.S.; Ziegler, C.J. The synthesis of dimeric Re-phenylenediimine conjugates: Spectroscopic and electrochemical studies. Eur. J. Inorg. Chem. 2014, 16, 2643-2652. [CrossRef]

16. Zhang, E.; Dai, X.; Zhu, Y.; Chen, Q.; Sun, Z.; Qiu, X.; Ji, X. Associating behavior of one polyimide with high molecular weight in solution through a relatively weak interaction. Polymer 2018, 141, 166-174. [CrossRef]

17. Semenov, N.A.; Kelbysheva, E.S.; Danilin, A.N. Electrorheological properties of suspensions of the polyimides based on 4,4'-diaminodiphenylsulfide. Mekhanika Kompozitsionnykh Materialov I Konstruktsii 2017, 23, 521-531.

18. Vinogradov, G.V.; Malkin, A.Y. Rheology of Polymers: Viscoelasticity and Flow of Polymers; Springer: Berlin/Heidelberg, Germany, 1980; ISBN 9783642522062.

19. Pakula, T. Polymer Rheology: Theory and Practice; Yanovsky, Y.G., Ed.; Chapman \& Hall: London, UK, 1993. [CrossRef]

(C) 2020 by the authors. Licensee MDPI, Basel, Switzerland. This article is an open access article distributed under the terms and conditions of the Creative Commons Attribution (CC BY) license (http://creativecommons.org/licenses/by/4.0/). 